\title{
Self-Healing Collagen-Based Hydrogel for Brain Injury Therapy
}

\author{
Raquel de la Cruz and David Díaz Díaz
}

\section{Contents}

1 Introduction

2 Collagen and Collagen Hydrogels

3 In Vitro Studies

4 In Vivo Studies

5 Concluding Remarks

References

\begin{abstract}
Hydrogels derived from biopolymers, also called biohydrogels, have shown potential for brain injury therapy due to their tunable physical, chemical, and biological properties. Among different biohydrogels, those made from collagen type I are very promising candidates for the reparation of nervous tissues due to its biocompatibility, noncytotoxic properties, injectability, and self-healing ability. Moreover, although collagen does not naturally occur in the brain, it has been demonstrated that collagen type I, which resides in the basal lamina of the subventricular zone in adults, supports neural cell attachment, axonal growth, and cell proliferation due to its intrinsic content of specific cell-signaling domains. This chapter summarizes the most relevant results obtained from both in vitro and in vivo studies using self-healing biohydrogels based on collagen type I as key component in the field of neuroregeneration.
\end{abstract}

R. de la Cruz and D. D. Díaz $(\bowtie)$

Departamento de Química Orgánica, Universidad de La Laguna, La Laguna, Tenerife, Spain

Instituto Universitario de Bio-Orgánica Antonio González, Universidad de La Laguna, La

Laguna, Tenerife, Spain

Institut für Organische Chemie, Universität Regensburg, Regensburg, Germany

e-mail: David.Diaz@chemie.uni-regensburg.de 
Keywords Brain therapy $\cdot$ Central nervous system $\cdot$ Collagen $\cdot$ Hydrogel $\cdot$ Regeneration

\section{Abbreviations}

2D

3D

BBB

bFGF

BMSC

CLG3/HLP

CNS

cRGD

CSPG

DGEA

DRG

ECM

EGF

ESC

FAK

FGF-2

FITC

FN

G3P

GDNF

GRP

HA

hMSC

LG3

LN

LP

MCAO

MSC

mTGase

NeuN

NGC

NGF

NPC

NSC

PC12

PCR

PNS

ReNcell

RGD

RGDT

SAPNS
Two dimensional

Three dimensional

Blood-brain barrier

Basic fibroblast growth factor

Bone marrow-derived mesenchymal stem cell

Collagen-binding LG3/histidine-tagged LP

Central nervous system

Cyclo(RGD-D-Phe-Val)

Chondroitin sulfate proteoglycans

Aspartic acid-glycine-glutamic acid-alanine

Dorsal root ganglion/ganglia

Extracellular matrix

Epidermal growth factor

Embryonic stem cell

Focal adhesion kinase

Fibroblast growth factor-2

Fluorescein isothiocyanate

Fibronectin

Glyceraldehyde 3-phosphate

Glial cell line-derived neurotrophic factor

Human glial-restricted precursor

Sodium hyaluronate (hyaluronic acid)

Human marrow stromal cell

Peptide LG3

Laminin

Peptide FNTPSIEKP

Middle cerebral artery occlusion

Mesenchymal stem cell

Microbial transglutaminase

Neural nuclear protein

Nerve guidance channel

Nerve growth factor

Neural progenitor cell

Neural stem cells

Pheochromocytoma cell

Polymerase chain reaction

Peripheral nervous system

Neural progenitor cell

Arginylglycylaspartic acid

Arginylglycylaspartic acid-threonine

Self-assembling peptide nanofiber scaffolds 
SC Schwann cell

SEM Scanning electron microscopy

\section{Introduction}

The central nervous system (CNS) is crucial for performing motor, sensory, and autonomic functions, including somatic injury repair. Unfortunately, the CNS has limited capacity for regeneration, resulting in the formation of glial scars and cysts. This makes the effects of neurotrauma, ischemia, hemorrhage, or neurodegenerative diseases displeasing and, very often, irreversible [1]. This is mainly due to the intrinsic properties of neural parenchyma (i.e., insufficiency of progenitor neural cells in the adult nervous system and slow ability of mature neural cells to regenerate, proliferate, and migrate [2]) and the heterogeneous microenvironment generated by the damage. This can lead to destruction of the blood-brain barrier (BBB), cytotoxicity (e.g., due to release of proteases and free radicals from necrotic cells), and trophic and oxygen deprivation.

Many therapeutic strategies designed for enhancing endogenous repair mechanisms of the nervous system have become unsuccessful due to the short half-life and systemic effects of injectable growth factors, as well as the poor survival, differentiation, and migration of transplanted stem cells. One of the most promising approaches to overcome these issues is the use of three-dimensional (3D) hydrogel networks with tunable physical and chemical properties [3]. In the context of brain tissue regeneration, such hydrogels must support both viscous flow under shear stress (shear-thinning during injection) and time-dependent recovery upon relaxation (self-healing after injection at the injury site) in order to achieve a minimally invasive surgery [4]. Moreover, many other features such as biocompatibility, biodegradability, porosity, cell adhesion ability, low cytotoxicity and immunogenicity, lack of mutagenicity, and lack of swelling, are also critical aspects that should be considered to develop hydrogel scaffolds suitable for clinical applications in neuroregeneration [5].

Hydrogels can serve as local transport systems for the delivery of drugs and signaling molecules specifically to the injury site and as 3D scaffolds providing appropriate physical support, substrates for cell adhesion, optimal nutrient and oxygen exchange, and protection to host and graft cells, thus facilitating extracellular matrix (ECM) formation [6]. Within this context, the main role of hydrogels in neuroregeneration is to exert control over the host neural tissue and grafted cell fate by sustaining attachment, neurite outgrowth, proliferation, migration, differentiation, and viability. Among many types of hydrogels, biohydrogels derived from preexisting components of body tissues, such as collagen, constitute promising biomaterials for brain injury therapy due to their biocompatibility, noncytotoxic properties, self-healing ability, and intrinsic content of cell-signaling domains that can efficiently promote cell growth [7].

Usually, biological materials such as collagen are turned into mechanically stable hydrogels by either cross-linking processes (i.e., chemical cross-linking, photochemical cross-linking, enzymatic cross-linking, thermal cross-linking) or mixing with other 
polymers. Moreover, in order to use these biomaterials in brain injury therapy, we should consider that neural progenitor cells (NPCs) receive instructive cues from chemical and physical sources which affect differentiation and growth. Therefore, conjugation of specific bioactive molecules/peptides onto the culture surface constitutes a good strategy to gain control on the signals that cells receive from external stimuli. In this sense, a logical design of materials for tissue engineering applications, including regeneration of damaged brain tissues, involves the incorporation of adhesive peptide sequences and cytokines into material constructs to promote cell penetration and host tissue integration by enabling cell-matrix and cell-cell interactions.

In this chapter, we summarize key results obtained in this field using self-healing biohydrogels based on collagen type I, including both in vitro and in vivo studies.

\section{Collagen and Collagen Hydrogels}

Collagen is the main structural protein found in the ECM of connective tissues in the body. It is the most abundant protein in mammals, and it represents approximately $30 \%$ of the whole-body protein content [8]. From a chemical point of view, collagens are trimeric molecules composed of three polypeptide $\alpha$-chains, which contain the repeating sequence $(\mathrm{G}-\mathrm{X}-\mathrm{Y})_{n}$, where $\mathrm{X}$ is normally proline and $\mathrm{Y}$ is hydroxyproline. Such repeating pattern allows the formation of a triple helix, so-termed tropocollagen, which is the most characteristic structural feature of the collagen protein family. It is well established that subsequent stabilization upon further packing of the tropocollagen subunits into fibrils eventually forms a 3D collagen hydrogel. There are 29 collagen types reported in the literature, which differ in size, structure, and functions [9].

Collagen [10] and hyaluronic acid (HA) [11] are the most common natural polymers in neural tissue engineering. In particular, it has been reported that collagen hydrogel scaffolds infused with nerve growth factor are capable of improving cell viability in vitro [12]. Moreover, neurons cultured in collagen hydrogels have been found to retain their capacity to generate spontaneous post-synaptic potentials, demonstrating functional synapse formation [13]. Importantly, collagen hydrogels without additional topographical features have also been used to treat spinal cord injury in rats in vivo. The results have showed that these gels are biocompatible, support axonal growth, and can improve limited functional recovery. In addition, collagen-based hydrogels have also been used to differentiate NPCs into neurons and glial cells and to support neurite outgrowth of CNS neurons [14].

Collagen type IV is widely presented in the adult nervous system where it forms basement membranes of the BBB and neuromuscular junctions, being also associated to neurogenesis in the embryonic and adult brain. Collagen type I (hereinafter referred to as collagen I) is one of the collagen types found in fractone, an extracellular matrix structure, which is present in the lateral wall of the ventricles. This is relevant because such location constitutes one of the main neural stem cell (NSC) niches in the adult brain [9]. As NSCs retain the ability to self-renew and produce the major cell types of the brain, they have been on the focus of restorative therapy for 
neurodegenerative disorders. Although collagen does not naturally occur in the brain, it has been proved that collagen I, which resides in the basal lamina of the subventricular zone in adults, supports neural cell attachment, axonal growth, proliferation, and guidance in neural development [15]. Collagen I also forms the dura mater (i.e., the thick membrane that surrounds the brain and spinal cord) and leptomeninges [16].

Collagen I is routinely obtained from rat tails, porcine, and bovine skin, and several research groups have demonstrated that the collagen source influences significantly the properties of the hydrogels that can be formed [17]. Due to its role in CNS development, as well as the self-healing capacity of its hydrogels, collagen $\mathrm{I}$ is considered to be a good candidate for brain tissue regeneration [15]. For instance, neural progenitor cells isolated from the rat embryonic CNS have been found proliferate and differentiated rapidly into both neurons and astrocytes in collagen I hydrogels [8]. Furthermore, the neurons in these hydrogels were found to develop neuronal features, including neuronal polarity, neurotransmitters, ion channels/receptors, and excitability. Several reviews have been reported in the literature regarding the use of different biopolymers to repair nerve injuries $[9,18]$.

\section{In Vitro Studies}

In 2007, Brannvall and co-workers developed a two-component collagen I-HA scaffold (1:1 volume mix) matrix to enhance the differentiation of mouse embryonic, postnatal, and adult NSCs and PCs (progenitor cells) [19]. The gel formation of the scaffold used in this study takes place during physiological conditions and is caused by the physical aggregation of collagen. The resulting network was found to retain hyaluronan and provided the stability required for culturing cells in $3 \mathrm{D}$. It is worth noting that the formation of mature neural cells from NSC/PC depends on its intrinsic machinery but also on external factors and signals derived from the ECM. Both components of the 3D matrix, when used separately, were found to be compatible with culture of cells from the nervous system. The authors demonstrated that their combination constitutes a favorable condition for neuronal differentiation of NSC/PC. By raising the temperature to $37^{\circ} \mathrm{C}$, gel formation of the collagen scaffold can be induced, and such property would be advantageous for tissue replacement after traumatic brain injury, where a cavity in the brain parenchyma is formed. By mixing cells with the scaffold at temperatures below $37^{\circ} \mathrm{C}$, the cellmatrix mixture may fill the cavity with ECM components and new cells. Specifically, stem cells isolated from different ages of CNS tissue were seeded in the presence of the 3D bio-scaffold and cultured in medium containing the mitogens epidermal growth factor (EGF) and fibroblast growth factor-2 (FGF-2), a condition that stimulates NSC/PC proliferation. In general, the results showed that progenitor cells from the embryonic brain had the highest proliferation rate, and adult cells the lowest, indicating a difference in mitogenic responsiveness. NSC/PC from postnatal stages downregulated nestin expression more rapidly than both embryonic and adult 
NSC/PC, indicating a faster differentiation process. In particular, after 6 days of differentiation in the 3D scaffold, NSC/PC from the postnatal brain had generated up to $70 \%$ neurons, compared with $14 \%$ in a two-dimensional (2D) culture. The reason for the relatively high percentage of apoptosis is most likely dead cells being trapped in the 3D scaffold, compared with 2D culture, in which dead cells are detached from the surface and washed out during medium exchange. Another contributing factor could be a difficulty with exchange of gases and nutrients into the core of the matrix, compared with the corresponding 2D culture, which is only one cell layer thick. However, NSC/PC from other ages gave rise to approximately the same proportion of neurons in 3D as in 2D (9-26\% depending on the source for NSC/PC). In the postnatal NSC/PC cultures, the majority of $\beta$ III-tubulin-positive cells expressed glutamate, g-aminobutyric acid, and synapsin I after 11 days of differentiation, indicating differentiation to mature neurons in the biocompatible hydrogel (Fig. 1).

Bozkurt and co-workers compared in vitro a cross-linked porcine collagen scaffold with a fibrin hydrogel-based system for the ability to support neurite outgrowth in the rat dorsal root ganglion (DRG) [20]. It is important to highlight that although DRG studies deal with the peripheral nervous system (PNS), they can provide valuable data for regeneration of the relevant adult neurons. The nerve guide described in this work showed a high degree of porosity and, more importantly, a remarkable degree of orientation, with channel sizes between 20 and $50 \mu \mathrm{m}$. Such collagen guidance channels were manufactured using a series of chemical and mechanical treatments with a patented unidirectional freezing process (Fig. 2). Hemisected rat DRGs were positioned such that neural and non-neural elements could migrate into the collagen scaffold (Fig. 3). After 21 days, S100-positive Schwann cells (SCs) migrated into the scaffold and aligned within the guidance channels in a columnar fashion, resembling "Bands of Büngner." Overall, the microstructural properties of collagen scaffold and the in vitro data after DRG loading make this scaffold a good candidate to be considered for promoting oriented nerve fiber regeneration in the PNS.

Around the same time, a study on DRG carried out by Blewitt and co-workers [21] revealed that hydrogels with low collagen concentrations $(0.4-1.0 \mathrm{mg} / \mathrm{mL})$ helped to achieve the longest neurite extension. Hydrogels with higher collagen concentration and thus higher stability required modifications with adhesive recognition peptides to exhibit similar properties. The aim of this study was to investigate the effects of inhibitory molecules on nerve growth in $3 \mathrm{D}$ environments as compared to $2 \mathrm{D}$ surfaces. Thus, soluble peptide sequences were used as competitive inhibitors of neurite extension in collagen gels. In order to determine the effect of collagen gel properties on neurite extension, dissociated DRG cells were seeded into an array of collagen gel concentrations. E9 chick dorsal root ganglion cells were seeded within collagen gels as well as onto collagen-coated glass and were exposed, for $24 \mathrm{~h}$, to one of three experimental peptide sequences, namely, arginine-glycine-aspartic acid-threonine (RGDT), cyclo(RGD-D-Phe-Val) (cRGD), or aspartic acid-glycineglutamic acid-alanine (DGEA) (Fig. 4). In 3D collagen gels, only the cRGD peptide sequence reduced neurite extension across a variety of gel concentrations. In contrast, on 2D surfaces, both RGD peptides reduced the number of cells expressing 
Self-Healing Collagen-Based Hydrogel for Brain Injury Therapy
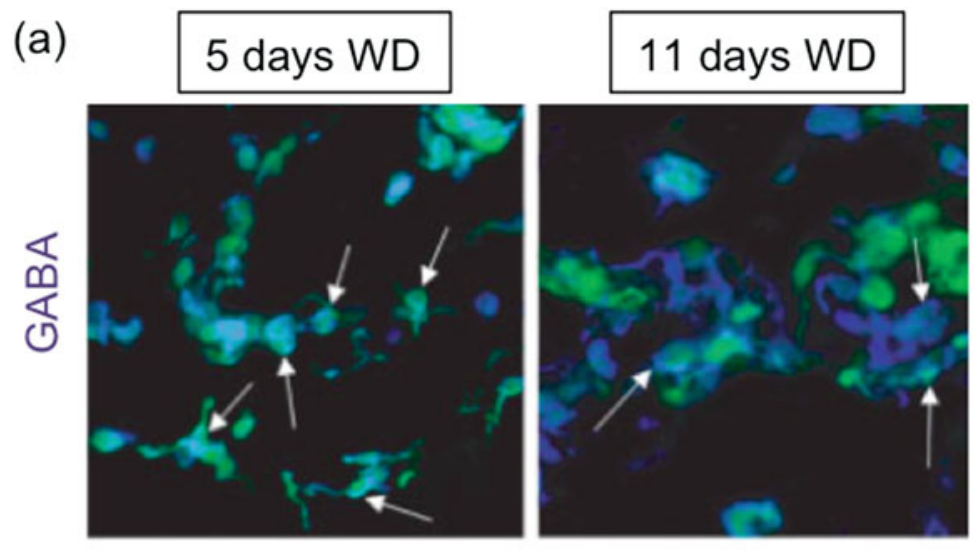

(b)
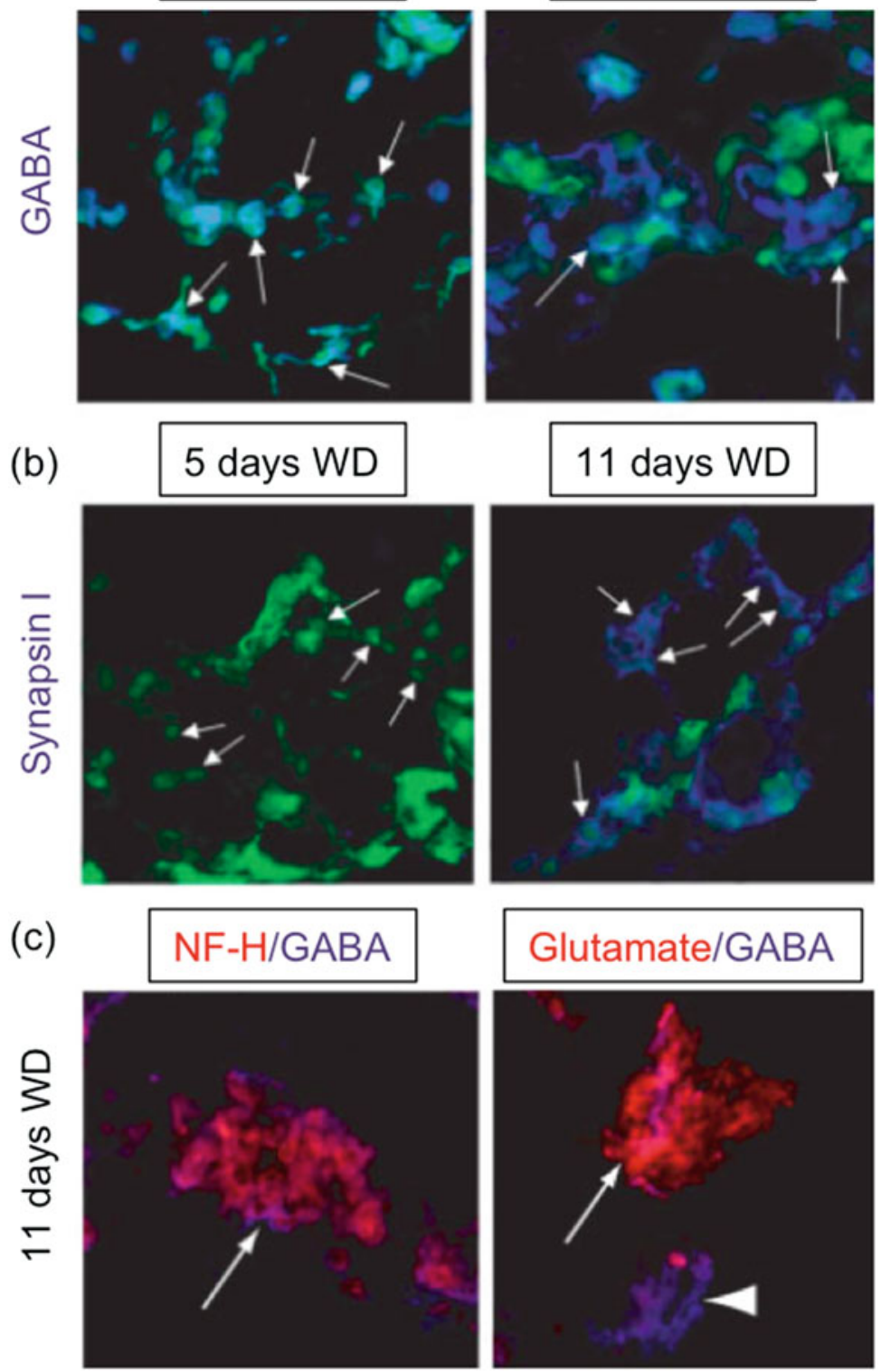

Fig. 1 P6 NS/PC grown in 3D express GABA, glutamate, and synapsin I upon growth factor withdrawal (WD). (a) GABA-positive neurons are formed after 5 days of $\mathrm{WD}$, and, after 11 days of WD, most bIII-tubulin-positive neurons are GABA immunoreactive. Signal from $\beta$ III-tubulin removed; arrows indicate neurons. 3,200. (b) Neurons expressing $\beta I I I-t u b u l i n$ are not synapsin I positive after 5 days of withdrawal. After 11 days of withdrawal, most $\beta$ III-tubulin-positive cells are synapsin I positive (blue). Signal from $\beta$ III-tubulin removed; arrows indicate neurons. 3,200. (c) After 11 days of withdrawal, mature neurons expressing NF-H (red) are expressing GABA (blue left, right arrowhead). Cells are also expressing glutamate (red right, arrow). Signal from EGFP has been removed. 3,200. Adapted with permission from reference [19], Copyright 2007 John Wiley $\&$ Sons 


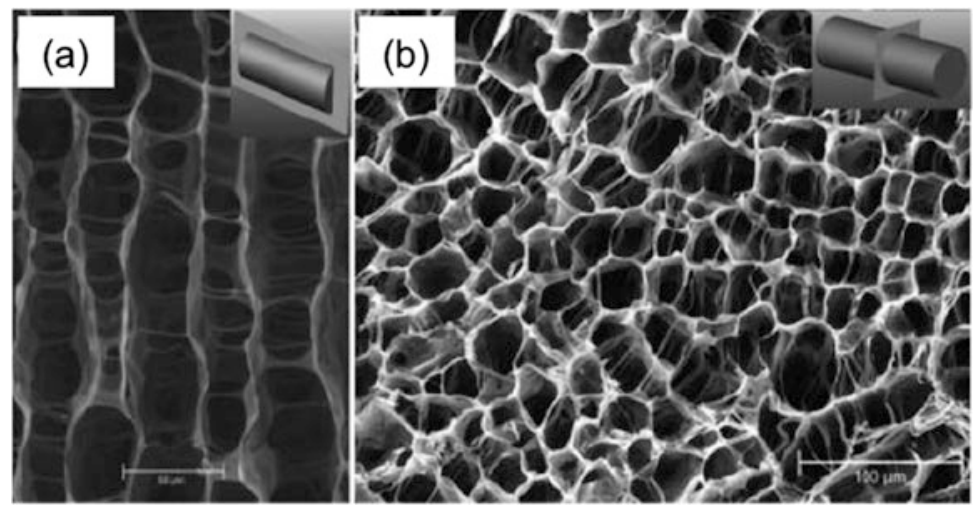

Fig. 2 Scanning electron microscopy (SEM) of the microscopic structure of the collagen matrix. Pore sizes ranged between 20 and $50 \mu \mathrm{m}$ (a, left, longitudinal section): extensive fenestrations interconnected the longitudinal guidance channels. (b, right, cross-section): Top view-longitudinal guidance channels pass from one end of the scaffold to the other. Scale bars (a) $50 \mu \mathrm{m}$ (b) $100 \mu \mathrm{m}$. The orientation of the sections is indicated by the schematic diagrams. Adapted with permission from reference [20]. Copyright 2007 Mary Ann Liebert

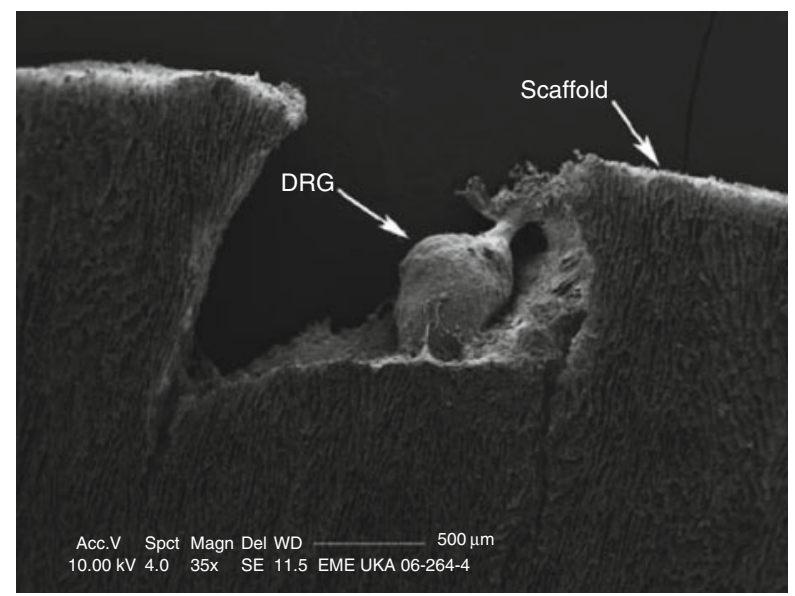

Fig. 3 Scanning electron microscopy (SEM) example of a dorsal root ganglion (DRG) loaded onto the collagen matrix. Scale bar $=500 \mu \mathrm{m}$. Adapted with permission from reference [20]. Copyright 2007 Mary Ann Liebert

neurites, but cRGD still exhibited superior inhibition of neurite expression (Fig. 5). Therefore, it seems that 2D substrate assessments for DRG growth do not accurately mimic growth in a 3D environment.

Deister and co-workers investigated neurite extension from explanted dorsal root ganglia cultured within co-gels made from laminin, fibronectin, collagen I, and HA [22]. In contrast to previous results, this research showed that apparently neither collagen nor HA concentration had effects on neurite outgrowth and length. The 


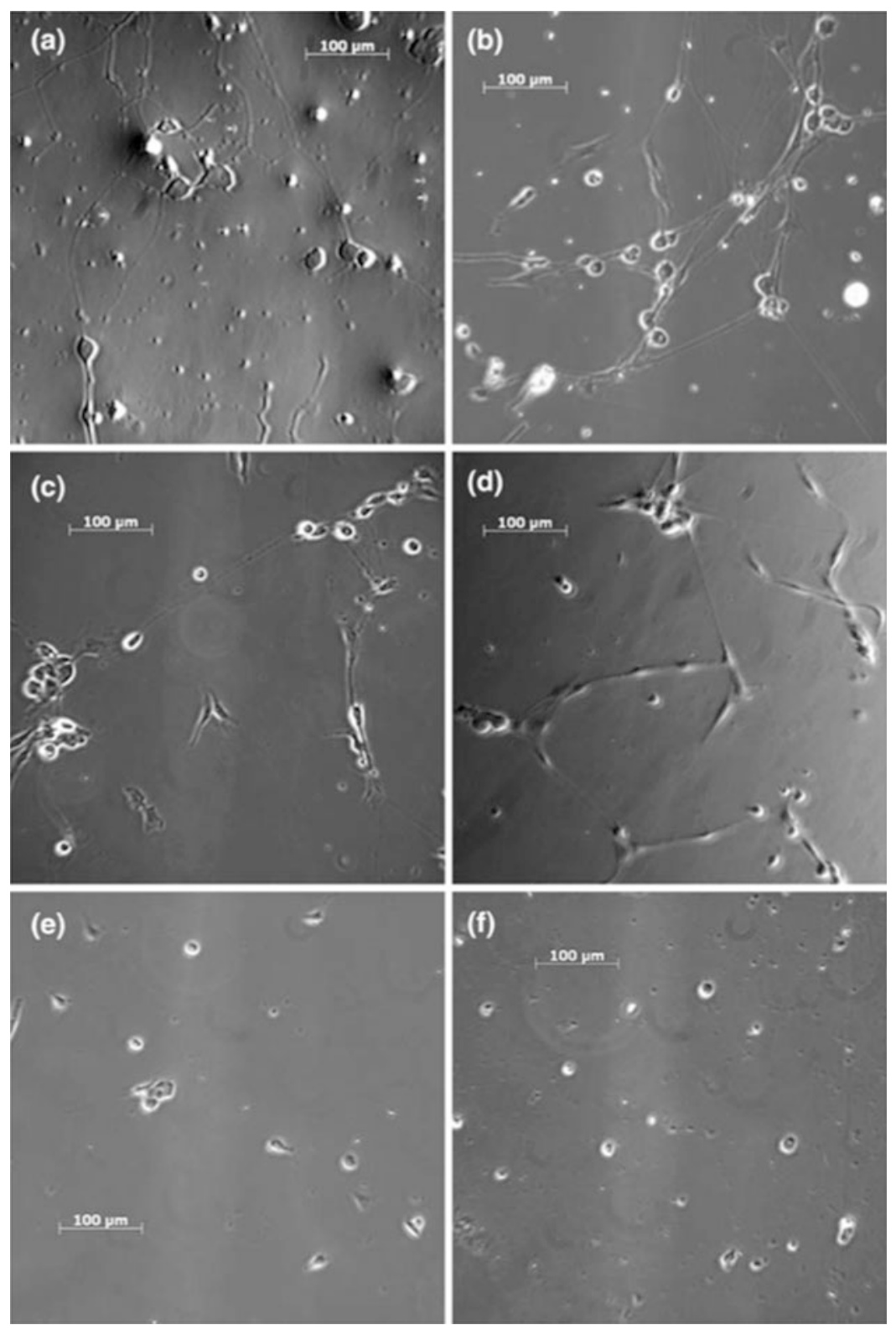

Fig. 4 Qualitative examination of cells on 2D substrates with various peptide treatments. All cultures were initially incubated $24 \mathrm{~h}$ (a) before peptide supplementation (imaged live). After subsequent $24 \mathrm{~h}$ incubation, cultures with RGES (c), DGEA (d), and no peptide added (b) exhibited similar networking and attachment. Cultures supplemented with RGDT (e) and cRGD (f) exhibited reduced networking and overall neurite expression. Reproduced with permission from reference [21]. Copyright 2007 Springer 


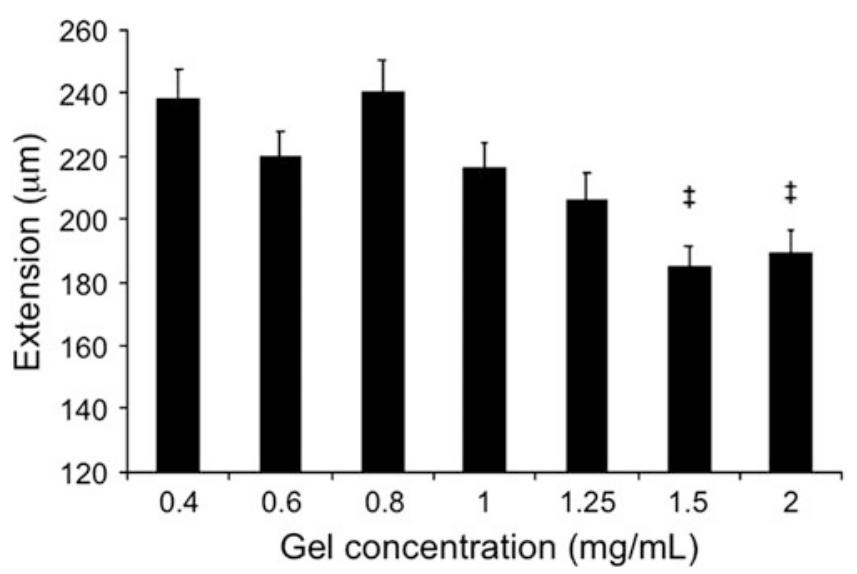

Fig. 5 Average neurite length after $24 \mathrm{~h}$ for each collagen gel concentration. Statistical differences (\$) were noted between lengths in 1.5 and $2.0 \mathrm{mg} / \mathrm{mL}$ gels and those in the range from $0.4-1.0 \mathrm{mg} /$ $\mathrm{mL}$. Error bars are SEM, $n \geq 100$ for each sample type. Adapted with permission from reference [21]. Copyright 2007 Springer

model used in this study used primary neurons that are actively involved in peripheral nerve injury. Briefly, the collagen I concentration of the hydrogels varied between 1 and $2.5 \mathrm{mg} / \mathrm{mL}$, and neurite outgrowth and length were analyzed, while hydrogels made with a lower collagen concentration $(0.5 \mathrm{mg} / \mathrm{mL})$ were found to be mechanically unstable to be used in these experiments. Laminin showed a strong, dose-dependent effect on both neurite length and outgrowth, whereas fibronectin displayed a slightly inhibitory effect on neurite extension. Similarly, the concentration of collagen I and HA showed no significant effects on neurite extension. Overall, the combinatorial effects observed among the four components of the formulations were additive rather than synergistic. The optimum co-gel formulation found in this study included $1.5 \mathrm{mg} / \mathrm{mL}$ of laminin and $1.5 \mathrm{mg} / \mathrm{mL}$ of collagen I (Fig. 6).

In 2009, Kofron and co-workers proceeded to guide neuritis using the surface patterning of a 3D collagen I hydrogel matrix with laminin (LN) and chondroitin sulfate proteoglycans (CSPG) [23]. LN and CSPG were chosen as representative molecules because LN is known to promote neuronal adhesion, migration, and neurite extension, while CSPG is an inhibitor of neuronal growth. By placing the DRG layer uniformly between collagen I hydrogel and either LN- or proteoglycan-covered glass, the authors confirmed laminin's micropatterning ability to guide DRG neurites on the surface of collagen (Fig. 7). In contrast, proteoglycan patterning did not lead to extension of DRG neurites. These results are relevant toward understanding how neurons integrate local structural and chemical cues to make net growth decisions.

In the same year, Hiraoka and co-workers investigated collagen-based hydrogel effects on rat fetal NSCs [24]. During this study, the authors demonstrated that the viability of neurosphere-forming cells embedded in a collagen hydrogel can be improved by incorporating a LN-derived cell-adhesive peptide involving glyceraldehyde 3-phosphate (G3P) in the hydrogel (Fig. 8). Because the viability of most cells 

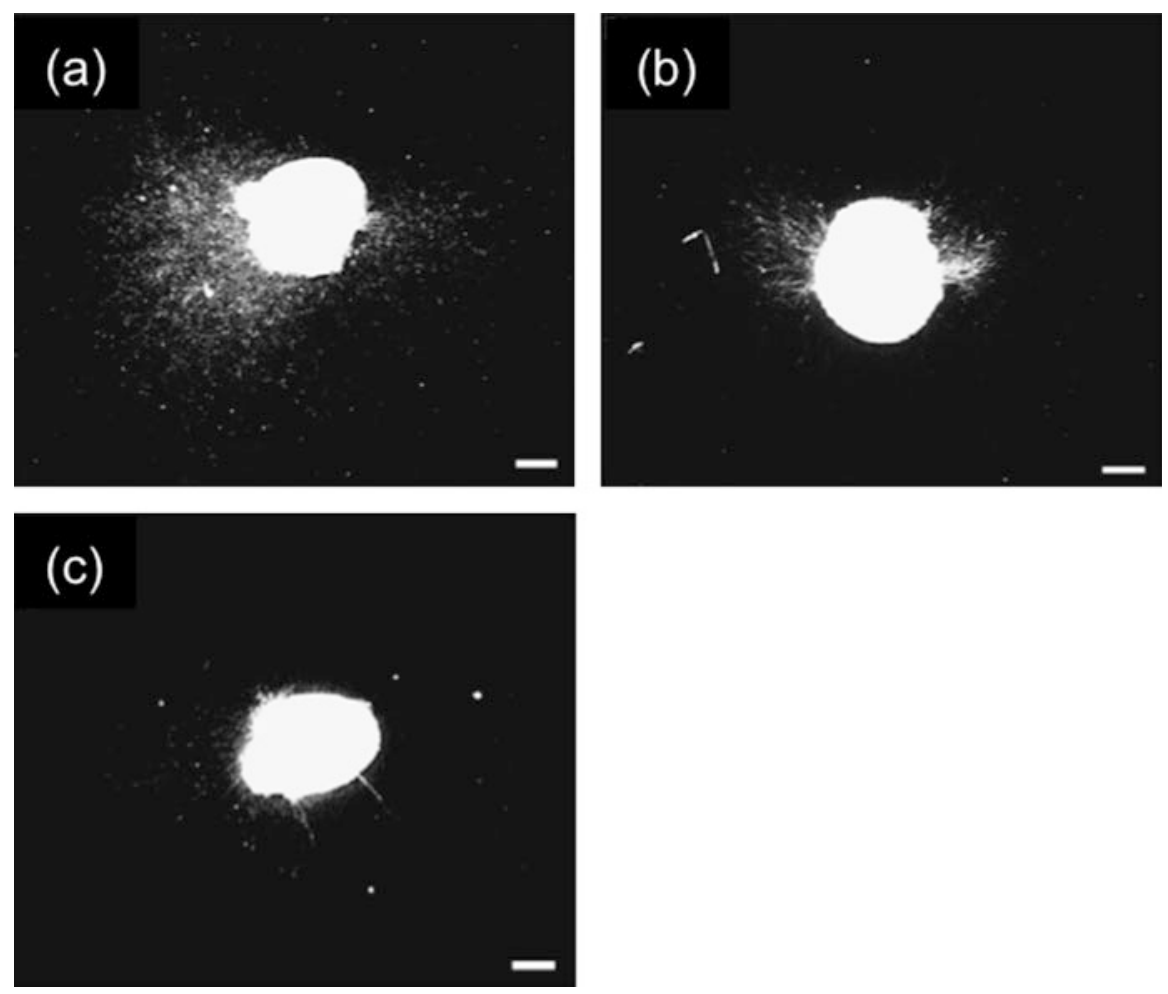

Fig. 6 Representative micrographs from DRGs cultured in gels made with (a) $1.5 \mathrm{mg} / \mathrm{mL}$ laminin and $1.5 \mathrm{mg} / \mathrm{ml}$ collagen $\mathrm{I}$, (b) $1.5 \mathrm{mg} / \mathrm{mL}$ collagen 1 only (control), and (c) $1.5 \mathrm{mg} / \mathrm{mL}$ fibronectin and $1.5 \mathrm{mg} / \mathrm{mL}$ collagen I. Scale bar is $200 \mu \mathrm{m}$. The laminin-containing gels (a) had significantly more neurite outgrowth and greater length than the collagen I only gel (b). The fibronectincontaining gels (c) had significantly less neurite outgrowth and shorter neurites than the collagen I only gel (b). Adapted with permission from reference [22]. Copyright 2007 Taylor \& Francis

largely relies on adhesion signaling, the improved viability is plausibly the consequence of enhanced cell adhesion in the hydrogel networks. G3P was originally identified by Kim and co-workers as a ligand for $\alpha 3 \beta 1$ integrin expressed on epithelial cells [25]. This peptide is contained in a G3 domain of a $\mathrm{LN} \alpha 3$ chain, a component of a LN-5 isoform. The interaction between LN-5 and $\alpha 3 \beta 1$ integrin was reported to have an antiapoptotic effect in a variety of epithelial cells [26]. Moreover, LN-5 is abundantly found in epithelial basement membranes [27] and also expressed in developing brain tissues [28]. Therefore, the collagen matrix bound with G3P as reported in this study can be regarded as a biomaterial that mimics in part the native basement membrane. Importantly, live/dead assays (Figs. 8 and 9) showed that a larger number of neurosphere-forming cells survived in collagen hydrogels containing G3P than in pure collagen hydrogels. The authors were unable to distinguish necrotic cells from apoptotic ones, and possibly both mechanisms were involved in observed cell death. Regarding necrotic conditions, if any, cells were equally affected in both hydrogels, and cell viability may be improved by optimizing culture conditions such as oxygen and 

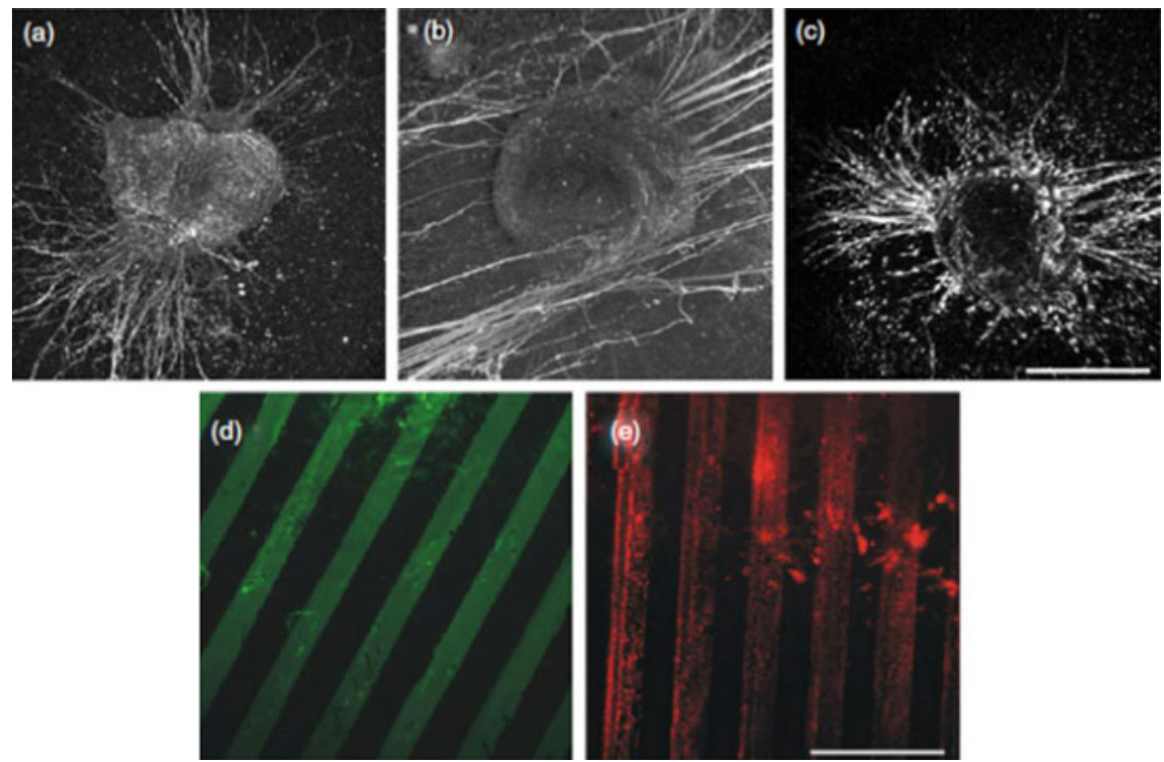

Fig. 7 In interface cultures, DRG explant growth patterns varied with interface cue. (a-c) 2D summations of maximum projections of a confocal stack of DRG explants grown for 3 days in interface cultures with no cue (a), micropatterned LN (b), or micropatterned CSPG (c) and stained for anti-neurofilament immunocytochemistry. (d, e) Coverslips removed from separate interface cultures with micropatterned LN (d) and micropatterned CSPG (e) and immunostained for anti-LN (d) and anti-CSPG (e). Top scale bar, $500 \mu \mathrm{m}$; bottom scale bar, $200 \mu \mathrm{m}$. Reproduced with permission from reference [23]. Copyright 2009 Institute of Physics Publishing

nutrient supply. For the observed difference in viability, the authors assumed that this was due to antiapoptotic signaling cascades activated in the cells in consequence of an interaction between G3P and $\alpha 3 \beta 1$ integrin. Indeed, integrin subunits $\alpha 3$ and $\beta 1$ are both expressed on the neurosphere-forming cells, being in good agreement with previous reports for neural stem cells [29], cerebral cortex neurons [30], and differentiating neuroblastoma cells [31].

In 2010 Yao and co-workers reported the ability of collagen I-based hydrogel to support neurite outgrowth in rat pheochromocytoma cells (PC12) [32]. Collagen scaffolds were prepared using different concentrations of a fluorescent labeledlaminin peptide (PPFLMLLKGSTR). Although the results of this study indicated that there is not a significant difference in neurite length on the LN-containing collagen hydrogel when compared with a native collagen scaffold, neurites showed a preferential growth orientation toward the high level of the LN peptide gradient on the collagen hydrogel (Fig. 10). This observation suggests the existence of different mechanisms involved in the regulation of neurite extension and neurite orientation.

In 2011, Lee and co-workers investigated the abilities of LN- and fibronectin (FN)-modified collagen to stimulate neuro-induction of rat BMSCs (bone marrowderived mesenchymal stem cells) [33]. The effects of the 3D gel conditions on the differentiation of MSCs into nerve cells were evaluated through observation of the 

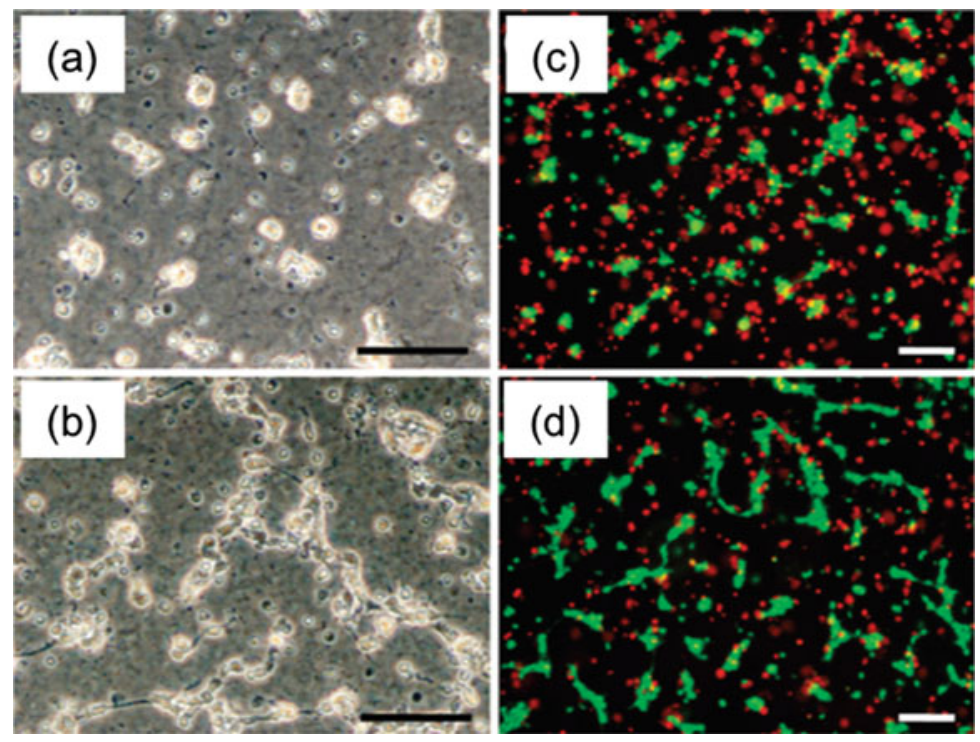

Fig. 8 Results of cell culture assays in collagen hydrogels with or without incorporated nG3PCBD. (a, b) Phase-contrast microphotographs of cells cultured for $48 \mathrm{~h}$ in (a) a pure collagen hydrogel and (b) a collagen hydrogel containing nG3P-CBD. Bars: $100 \mu \mathrm{m}$. (c, d) Fluorescent microphotographs of cells cultured for $48 \mathrm{~h}$ in (c) a pure collagen hydrogel and (d) a collagen hydrogel containing nG3P-CBD. Cells were stained with calcein-AM (live cells in green) and propidium iodide (dead cells in red). Bars: $100 \mu \mathrm{m}$. Adapted with permission from reference [24]. Copyright 2009 The American Chemical Society

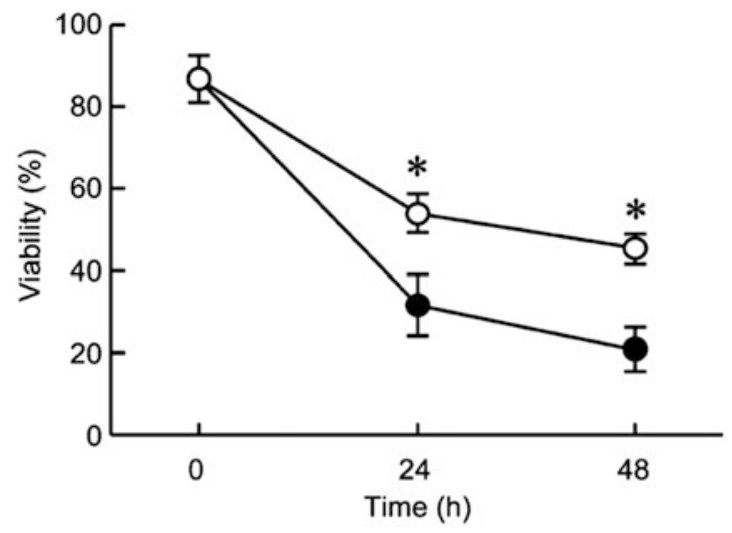

Fig. 9 The viability of cells cultured for 24 and $48 \mathrm{~h}$ in (open circle) collagen hydrogels containing nG3P-CBD or (filled circle) pure collagen hydrogels. Data are expressed as mean \pm standard deviation for $n=4$. $*$ Statistically significant ( $p<0.05$, Tukey's HSD test). Reproduced with permission from reference [24]. Copyright 2009 The American Chemical Society 


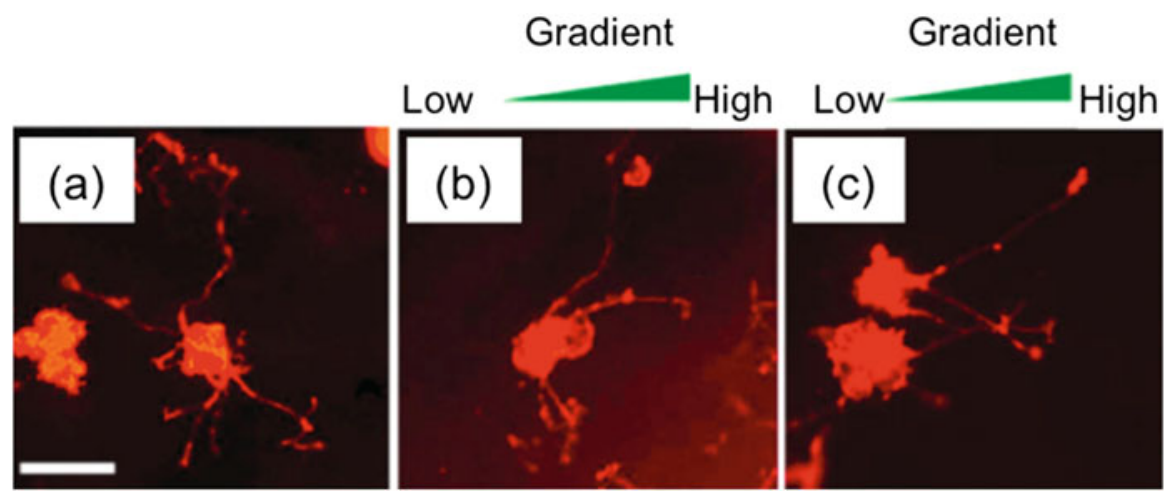

Fig. 10 Neurite growth on collagen scaffold with laminin peptide gradient. (a) Neurite growth on collagen scaffold without laminin peptide gradient. Neurite growth on (b) native collagen scaffold and on (c) cross-linked collagen scaffold with laminin peptide gradient. Scale bar: $100 \mu \mathrm{m}$. Adapted with permission from reference [32]. Copyright 2010 Wiley Periodicals

cellular morphology and protein and molecular assays. The adhesive proteincontaining hydrogels induced neuronal cell differentiation of BMSCs without the use of chemical differentiation factors, presumably due to the low stiffness of the 3D collagen microenvironment mimicking native brain tissue. The principal fate of MSCs is self-renewal without amplification and/or differentiation when cultured on a normal 2D culture dish without a supply of neurogenic medium, such as nerve growth factor (NGF), and this was also observed in the present study. Immunofluorescence staining, Western blot, and fluorescence-activated cell sorting analyses demonstrated that a large population of cells was positive for neural nuclear protein $(\mathrm{NeuN})$ and glial fibrillary acidic protein, which are specific to neuronal cells, when cultured in the 3D collagen hydrogel. The dependence of the neuronal differentiation of MSCs on the adhesive proteins containing 3D gel matrices is considered to be closely related to focal adhesion kinase (FAK) activation through integrin receptor binding, as exposed by an experiment showing no neuronal outgrowth in the FAK knockdown cells and stimulation of integrin b1 gene.

In 2012, Swindle-Reilly studied dissociated E9 chick DRG cells in a collagen I hydrogel modified with $\mathrm{LN}$ at concentrations of $0,1,10$, or $100 \mu \mathrm{g} / \mathrm{mL}$ [34]. This study revealed that low-concentrated unmodified collagen hydrogels $(0.4-1.5 \mathrm{mg} / \mathrm{mL})$ supported a typical bimodal neurite growth more than the LN-modified variant. Expression of integrin subunits, $\alpha 1, \alpha 3, \alpha 6$, and $\beta 1$, was confirmed by polymerase chain reaction (PCR) and immunolabeling in the $3 \mathrm{D}$ scaffolds. As expected, the increase in collagen concentration increased the stiffness of the gels (Fig. 11). Although confocal microscopy showed LN following the collagen fibers (Fig. 12), the addition of LN caused minimal changes on the stiffness of the scaffolds at any concentration of collagen.

In 2013, Koutsopoulos and co-workers compared viability and differentiation of the mouse adult NSCs in peptide nanofiber hydrogels, Matrigel ${ }^{\mathrm{TM}}$, and collagen [35]. Preliminary studies performed by the same group showed that self-assembling peptides support neuronal cell attachment and extensive neurite outgrowth and 


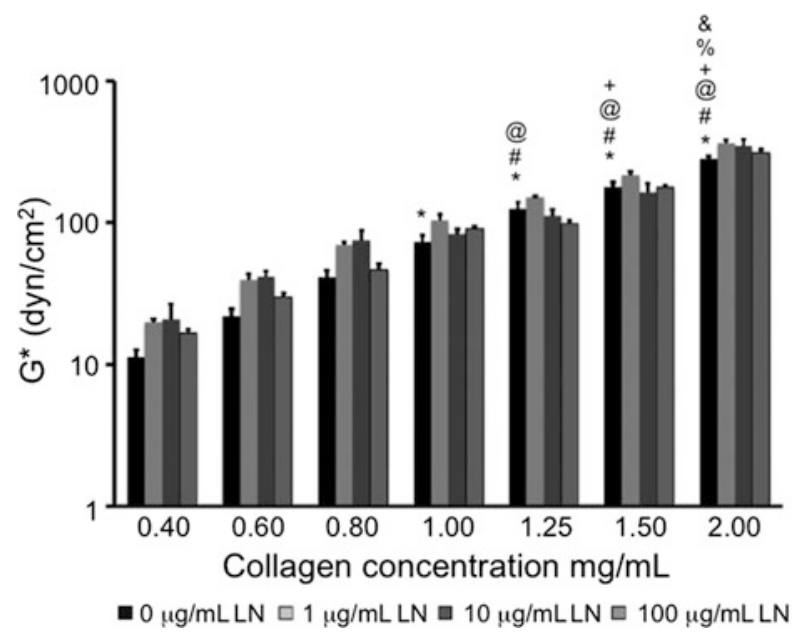

Fig. 11 Mechanical stiffness increases with increasing concentration of collagen; however, the addition of LN did not alter the stiffness. Mechanical stiffness of collagen gels with added LN was measured by the overall modulus, $G *$ with oscillatory shear rheometry within the linear viscoelastic range. Significance $(p<0.05)$ is noted by the symbols for plain gels only: $*$ compared to $0.4 \mathrm{mg} \mathrm{mL}{ }^{-1}$ collagen gels, \# compared to $0.6 \mathrm{mg} \mathrm{mL}^{-1}$ collagen gels, @ compared to $0.8 \mathrm{mg} \mathrm{mL}^{-1}$ collagen gels, + compared to $1.0 \mathrm{mg} \mathrm{mL}^{-1}$ collagen gels, $\%$ compared to $1.25 \mathrm{mg} \mathrm{mL}^{-1}$ collagen gels, and \& compared to $1.5 \mathrm{mg} \mathrm{mL}^{-1}$ collagen gels. No differences were noted with added $\mathrm{LN}$ within any concentration of collagen. Adapted with permission from reference [34]. Copyright 2012 Institute of Physics Publishing

promote active and functional synapse formations when neural cells were plated on the surface of preformed peptide hydrogel matrices. In this research, neural stem cells from the subventricular zone of an adult mouse were used. To stimulate extensive self-renewal of the neural stem cell population, human FGF-2, human EGF, and insulin in the stem cell propagating medium were also added. The authors found that continuous provision of both FGF-2 and EGF was necessary to sustain nonadherent neurosphere formation of neural stem cells. Tissue cultures in collagen I hydrogel were characterized by low cell migration, cell clustering, and limited neural stem cell differentiation. This may be due to the biological incompatibility between neural cells and collagen I, a substance not found in mammalian brain tissue. It is known that neural cells (especially differentiated neurons and immortalized neural cells) survive in collagen I in the presence of a cocktail of growth factors. Nevertheless, such studies are performed for short periods of times (i.e., up to 2 weeks), and they are characterized by low cell migration and differentiation. When neural tissue cultures were investigated for longer periods of time, the authors found enhanced cell survival rates in peptide nanofiber hydrogels compared to Matrigel ${ }^{\mathrm{TM}}$ and collagen I, suggesting that the beneficial effect of Matrigel ${ }^{\mathrm{TM}}$ on tissue cultures is mainly due to non-quantified growth factors and cytokines that are present in the material (Fig. 13). It is clear that when these chemical stimuli are removed, the material itself is not suitable to support a viable neural tissue culture. This is yet 
(a)
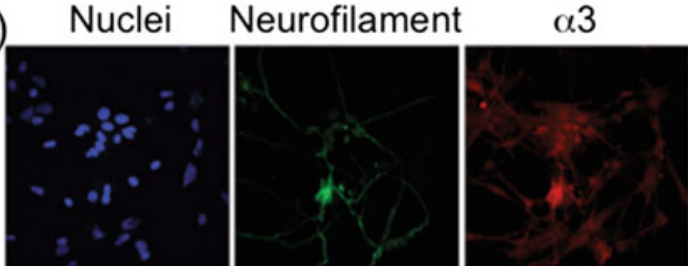

Merge

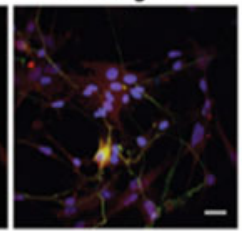

2D Lamini

(b)

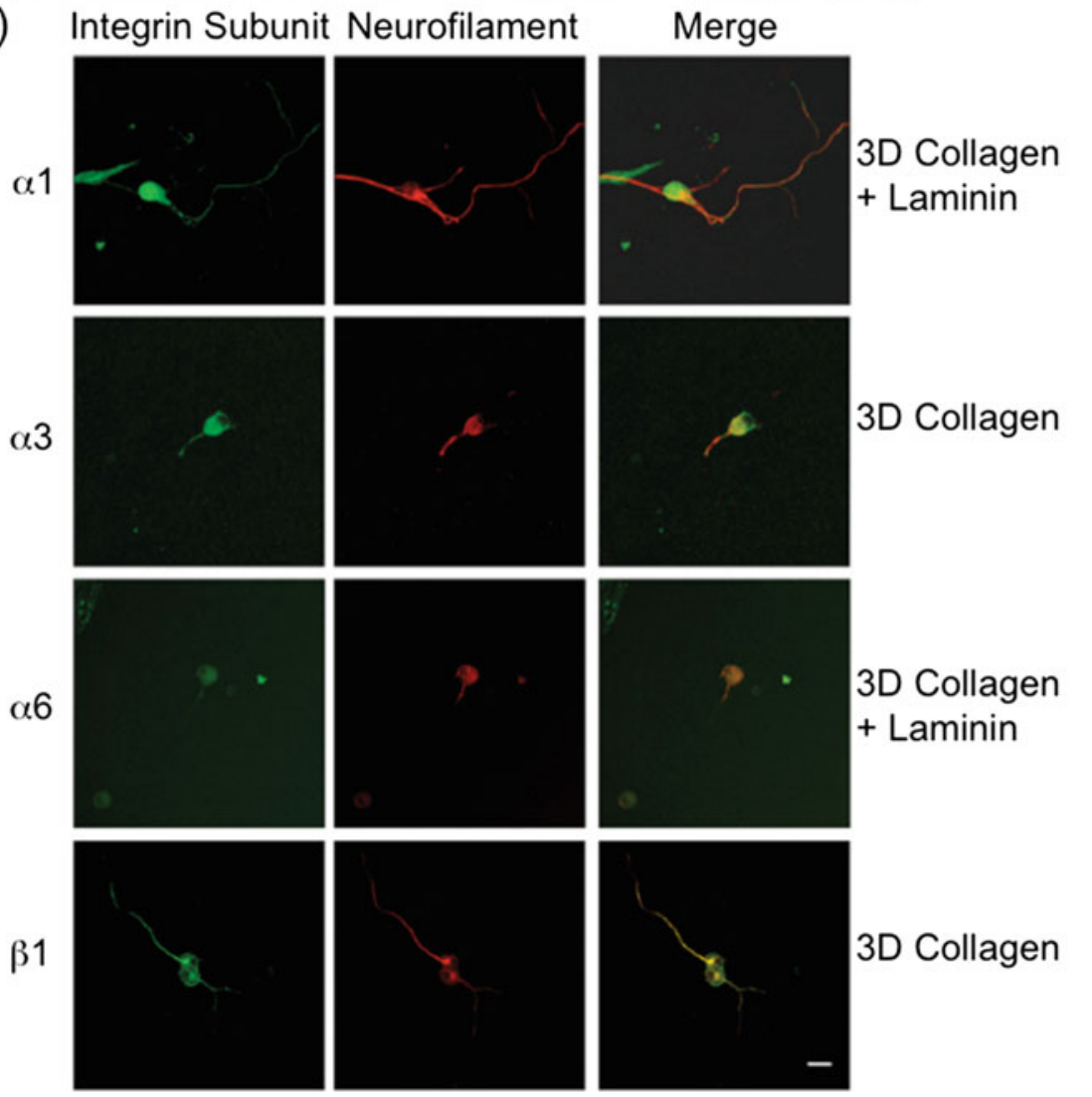

Fig. 12 Representative confocal images for DRG neurons growing on a 2D LN surface (a) or within 3D collagen $\left(1 \mathrm{mg} \mathrm{mL}^{-1}\right)$ gels with or without $100 \mu \mathrm{g} \mathrm{mL}^{-1} \mathrm{LN}$ (b) are shown. In (a), the neurons growing on the $\mathrm{LN}$ surface were stained for $\alpha 3$, neurofilament, and nuclei, with the merge shown on the right. The images show that the $\alpha 3$ subunit is expressed within neurons and glial/ fibroblast support cells. In (b), neurons growing within the 3D scaffolds were stained with antibodies that are specific to the integrin receptor subunits, $\alpha 1, \alpha 3, \alpha 6$, and $\beta 1$ and neurofilament antibody specific for neurons, with the merge shown on the right. Scale bar is $20 \mu \mathrm{m}$. Adapted with permission from reference [33]. Copyright 2012 Institute of Physics Publishing

another indication of the important role of the matrix environment in inducing neural stem cells into diverse differentiation pathways. In addition, rheological measurements of the peptide hydrogels, Matrigel ${ }^{\mathrm{TM}}$, and collagen I were performed to determine their mechanical properties. Matrigel ${ }^{\mathrm{TM}}$ and collagen I concentrations 
Fig. 13 Cell survival as a function of time. Live/dead assay of 3D neural cell tissue cultures in modified peptides shows increased cell survival compared to the unmodified ac-(RADA) $4^{-}$ $\mathrm{CONH}_{2}$ hydrogel,

Matrigel $^{\mathrm{TM}}$, and collagen

I. Adapted with permission from reference

[35]. Copyright 2013

Elsevier

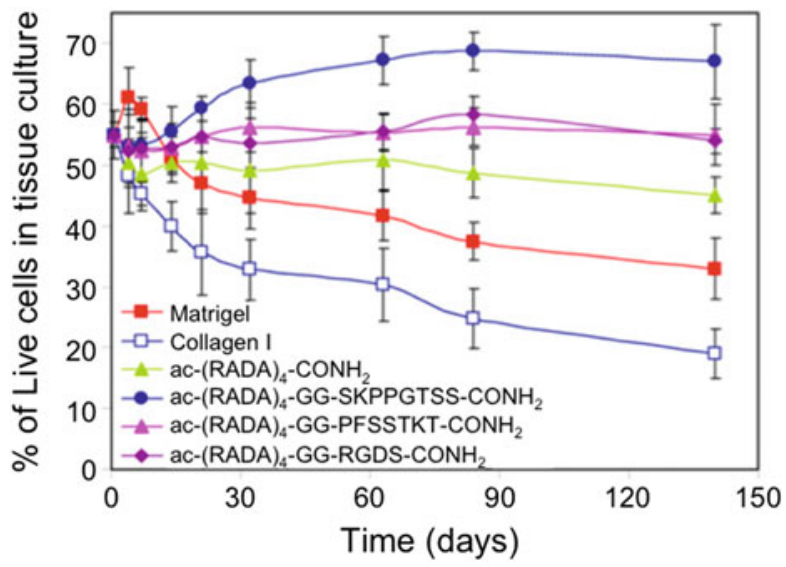

used in these experiments resulted in hydrogels with flow properties resembling those of the peptide hydrogels. Therefore, differences in cell behavior in different matrices could not be due to differences in the mechanical properties of the hydrogels.

\section{In Vivo Studies}

Despite the promising results reported so far in in vitro studies, it is also important to determine whether these results correlate with what occurs in in vivo conditions. In this context, $\mathrm{Yu}$ and co-workers have used tissue engineering to implant transient MCAO (middle cerebral artery occlusion) rats with commercially available biodegradable 3D porous collagen I sponges containing rat embryonic NSCs to develop a therapy for cerebral ischemic injury [36]. As expected, the results obtained in this study confirmed that collagen has good cell and tissue compatibility. Indeed, its long-term safety, stability, and efficacy in vivo have been adequately established in humans [37]. Experimentally, NSCs from E14 d rats were dissociated and cultured by neurosphere formation in serum-free medium in the presence of basic fibroblast growth factor (bFGF), then seeded onto collagen to measure cell adhesive ability. Wistar rats $(n=100)$ were subjected to $2 \mathrm{~h}$ middle cerebral artery occlusion. After $24 \mathrm{~h}$ of reperfusion, rats were assigned randomly to five groups: NSCs-collagen repair group, NSCs repair group, unseeded collagen repair group, MCAO medium group, and sham group. Neurological, immunohistological, and electronic microscope assessments were performed to examine the effects of these treatments. Scanning electronic microscopy (SEM) showed that NSCs assembled in the pores of collagen. At 3, 7, 15, and 30 days after transplantation of the NSC-collagen complex, some of the engrafted NSCs survive, differentiated, and formed synapses in the brains of rats subjected to cerebral ischemia. Six days after transplantation of the NSC-collagen complex into the brains of adult ischemic rats, the collagen gel 
began to degrade, and 30 days after transplantation, the collagen had degraded completely. Herein, it was concluded that the beneficial effect of the complexes is mediated by a neuroprotective rather than a regenerative mechanism.

In the same year, Zhong and co-workers carried out a study in a photothrombotic stroke murine model [38]. The stroke cavity provides an ideal target for transplantation because it is a compartmentalized region of necrosis, can accept a high volume transplant without tissue damage, and lies directly adjacent to the most plastic brain area in stroke. However, direct transplantation into the stroke cavity usually causes massive death in the transplant. To overcome these limitations, the authors tested stem/progenitor transplants within a specific cross-linked biohydrogel matrix made of hyaluronan, heparin, and collagen I to create a favorable environment for transplantation into the infarct cavity after stroke, and the results were compared to those obtained from direct injection of stem cells without hydrogel support. In this study, both embryonic cortex-derived NPCs and ESC (embryonic stem cell)-derived NPCs were used. Overall, hyaluronan-heparin-collagen hydrogels were found to promote the survival of NPCs derived from both the fetal cortex and ESCs, with no effect on cellular differentiation or migration of the cells from the stroke site. Quantitative analysis of the transplant and surrounding tissue indicates diminished inflammatory infiltration of the graft with the hydrogel transplant. In this respect, the beneficial effect of the hydrogel was found to be at least twofold higher than that obtained with NSCs in the absence of the gel matrix.

In 2013, Hoban and co-workers provided evidence for collagen I as a noncytotoxic and self-healing hydrogel in situ [39]. The researchers injected the striatum of sham rats with glial cell line-derived neurotrophic factor (GDNF)overexpressing rat bone marrow MSCs (GDNF-MSCs) encapsulated in a collagen hydrogel cross-linked with 4S-StarPEG (PEG ether tetrasuccinimidyl glutarate) (Fig. 14). In vitro studies confirmed that the collagen I hydrogel was nontoxic to neural cells or MSCs seeded within it and also permitted diffusion of GDNF from GDNF-MSCs into the cell culture medium. More importantly, it significantly reduced the host brain's response to the cells by reducing the recruitment of both microglia and astrocytes at the site of delivery. Although the hydrogel prevented micro- and macrogliosis compared to the medium-injected control group, the scaffold poorly supported MSCs survival and decreased in volume several days post gelation both in vitro and in vivo.

At the same time, Liang and co-workers developed a set of injectable hydrogels with variable HA:gelatin:PEG diacrylate ratios and used them to encapsulate C17.2 NSCs (mouse immortalized NSCs), human ReNcells (human immortalized NPCs), and human GRPs (glial-restricted progenitor cells) [40]. The gelation properties of the hydrogel were first characterized and optimized for intracerebral injection, resulting in a 25 min delayed injection after mixing the hydrogel components. To determine the optimal time for hydrogel delivery into the brain, the authors compared the resulting morphology of the hydrogel scaffold in vivo following an immediate or delayed injection. It was found that delayed injection produced optimal results, without leakage of gelatin into the brain tissue. The authors showed that an increase in gelatin content extended the gelation time, while HA promoted survival of all cell lines. In contrast, 

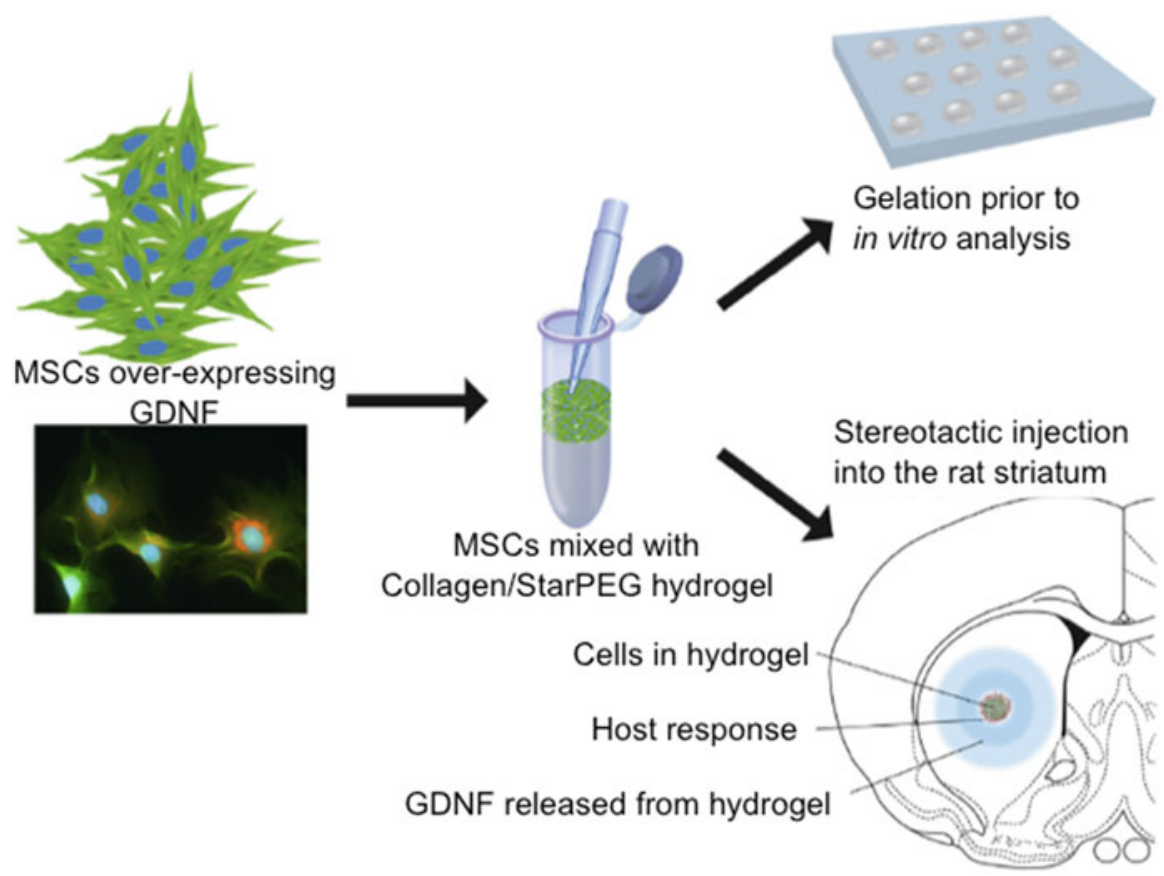

Fig. 14 Overall design of the study. Bone marrow-derived MSCs were extracted from the femora and tibiae of GFP transgenic Sprague-Dawley rats and transduced to overexpress human GDNF using a murine leukemia virus (in the photomicrograph, GDNF and nuclei are stained in red (immunofluorescence) and blue (DAPI), respectively). These were suspended in a type 1 collagen hydrogel prepared from bovine Achilles tendon, which was kept on ice to prevent gelation. The cellseeded collagen hydrogel was then subjected to a number of in vitro validation studies (i.e., impact of the hydrogel on astrocyte viability, MSC viability, and GDNF release) which were followed by in vivo studies in the adult rat brain to determine the host's response to the hydrogel and the impact of the hydrogel on the survival of, GDNF release from, and the host response to the GDNF-MSCs. In the in vivo studies, the striatum was infused bilaterally at coordinates $\mathrm{AP}=0.0, \mathrm{ML} \pm 3.7$ (from bregma) and DV -5.0 below dura. Adapted with permission from reference [39]. Copyright 2013 Elsevier

gelatin promoted survival and proliferation only in ReNcells and C17.2 cells. The hydrogels suppressed the innate immune response and improved viability of ReNcells injected into the striatum of immunodeficient mice. Interestingly, it was also found that hydrogel implantation can evoke a hitherto unreported immune response in the brain of immunocompetent animals. Since its innate immune system is still intact, activation of microglia and infiltrating leukocytes into the needle track may still be found in the control animals. In contrast, only a mild response occurred in the hydrogel scaffold groups. These findings suggest that the hydrogel suppresses the innate immune response induced by implanted human cells.

Nakaji-Hirabayashi and co-workers also studied a collagen hydrogel with or without laminin-derived peptides with rats in vivo [41]. Poor viability of cells 
transplanted into the brain has been a critical problem associated with stem cellbased therapy for Parkinson's disease. To overcome this problem, a collagen hydrogel incorporating an integrin-binding protein complex was prepared and used as a carrier for neural stem cells. The protein complex consisted of two polypeptides containing the G3 domain of a laminin $\alpha 1$ chain and the C-terminal oligopeptide of a laminin $\gamma 1$ chain. These polypeptides were fused with $\alpha$-helical segments, which spontaneously formed a coiled-coil heterodimer and with the collagen-binding peptide that facilitated the binding of the heterodimer to collagen networks. Through in vivo studies, the authors demonstrated that infiltration of microglial cells was physically blocked by the collagen hydrogel and the apoptotic cell death could be minimized by ligating integrins with the collagen-binding LG3 (CLG3)/histidine-tagged LP (HLP) complex incorporated in the collagen hydrogel. Accordingly, cell viability was significantly improved at the early stage after transplantation into the striatum (Fig. 15). This effect is caused by two antiapoptotic effects: integrin ligation and the suppression of microglial infiltration.

It is considered that the inflammatory response in the brain is caused by microglia activated by foreign substances such as graft cells. The activated microglia infiltrates

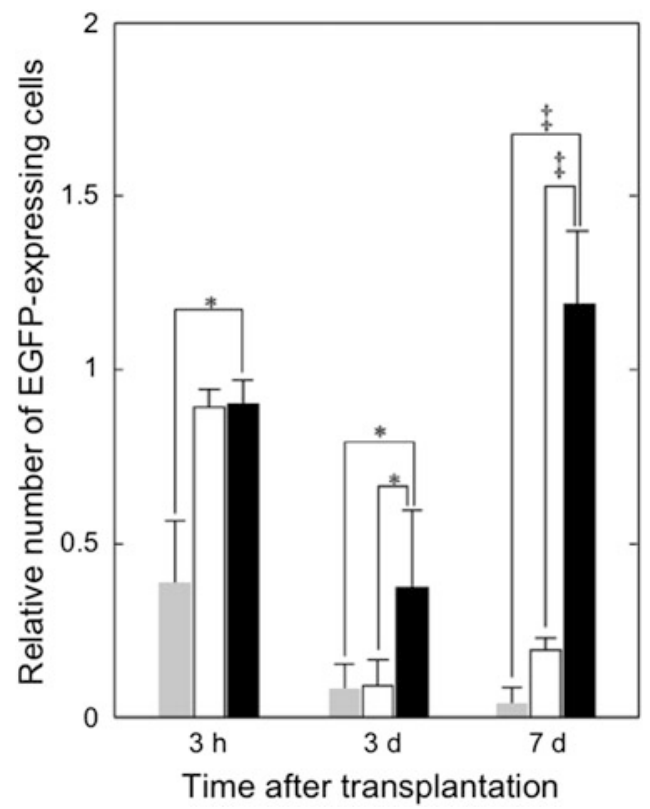

Fig. 15 Number of living cells determined 3 h, 3 days, and 7 days after transplantation into the rat brain. Cells were suspended in collagen hydrogels (closed bar) with or (open bar) without the CLG3/HLP complex, or (hatched bar) in medium. The number of EGFP-expressing cells relative to that of transplanted cells $\left(1.5 \times 10^{6}\right.$ cells $)$ was determined, and the data are expressed as the mean \pm standard deviation (day $0, n=3$; day $3, n=10$; day $7, n=9$ ). The symbols $*$ and $\$$ indicate statistical significance (Tukey's HSD test, $* p<0.05$ and $\$ p<0.01$ ). Adapted with permission from reference [41]. Copyright 2013 The American Chemical Society 
into the graft regions to release various cytotoxic cytokines. It was previously reported that the use of materials such as hydrogels would be beneficial to the protection of transplanted cells from inflammatory responses in the brain. NakajiHirabayashi's results demonstrated that the infiltration of microglia is inhibited at the early stage after transplantation, most likely due to the effect that the collagen hydrogel acts as a physical barrier against microglial infiltration. Although a collagen hydrogel itself may also initiate inflammation, it was speculated that this is overwhelmed by the effect of isolating graft cells from microglia.

\section{Concluding Remarks}

In conclusion, taking advantage of their tunable physical, chemical, and biological properties, 3D biohydrogels made of collagen type I have shown great potential for brain injury therapy. Among other features, these hydrogels are biocompatible, noncytotoxic, and self-healable after injection, which is a major requirement for in vivo applications in neuroregeneration. Although collagen does not naturally occur in the brain, it has been demonstrated that collagen type I, which resides in the basal lamina of the subventricular zone in adults, supports neural cell attachment, axonal growth, and cell proliferation due to its intrinsic content of specific cellsignaling domains.

The results derived from the studies summarized in this chapter illustrate the importance of several aspects such as (1) the necessary optimization of cell systems before attempting cell replacement in vivo, (2) the combination of the gel matrix with adhesive peptide sequences and proteins with appropriate cell attachment sites, (3) the use of SCs and their alignment within the guidance channels for the regeneration process, and (4) the appropriate stiffness, growth factor sequestering ability, and injectability feature of the biohydrogel system.

Despite the promising results obtained during the last decade in both in vitro and in vivo models, more detailed mechanistic studies are still necessary to understand the function of collagens in the brain, which should be correlated to the effects obtained during the use of their hydrogels in brain therapy. For instance, additional studies are still necessary to visualize the interactions of collagens with other ECM molecules, cell surface receptors, and downstream signaling pathways, which is critical to understand the underlying connection between the presence of collagen and certain pathogenesis in the nervous system. Furthermore, future studies involving specific functionalization of the collagen hydrogel would be important to release on demand biological cues, glial scar modulating enzymes, or pro-survival factors for in vivo therapy. In addition, advances in microfabrication techniques will likely expand the use of biohydrogel scaffolds in neuroregeneration. In any event, any new developed collagen-based hydrogel should be thoroughly tested for immuno-/ allergenicity prior to their use in clinical applications. 
Acknowledgments D.D.D. thanks the University of Regensburg (Germany) and the University of La Laguna (Spain) for financial support and the Ministry of Science, Innovation and Universities (Spain) for the Senior "Beatriz Galindo" Distinguished Researcher Award.

\section{References}

1. Struzyna LA, Katiyar K, Cullen DK (2014) Living scaffolds for neuroregeneration. Curr Opin Solid State Mater Sci 18:308-318. https://doi.org/10.1016/j.cossms.2014.07.004

2. Anderson MA, O'Shea TM, Burda JE, Ao Y, Barlatey SL, Bernstein AM et al (2018) Required growth facilitators propel axon regeneration across complete spinal cord injury. Nature 561:396-400. https://doi.org/10.1038/s41586-018-0467-6

3. Carballo-Molina OA, Velasco I (2015) Hydrogels as scaffolds and delivery systems to enhance axonal regeneration after injuries. Front Cell Neurosci 9:1-12. https://doi.org/10.3389/fncel. 2015.00013

4. Niemczyk B, Sajkiewicz PŁ, Kolbuk D (2018) Injectable hydrogels as novel materials for central nervous system regeneration. J Neural Eng 15:51002. https://doi.org/10.1088/17412552/aacbab

5. Orive G, Anitua E, Pedraz JL, Emerich DF (2009) Biomaterials for promoting brain protection, repair and regeneration. Nat Rev Neurosci 10:682-692. https://doi.org/10.1038/nrn2685

6. Zhang YS, Khademhosseini A (2017) Advances in engineering hydrogels. Science 356: eaaf3627. https://doi.org/10.1126/science.aaf3627

7. Peppas N (2006) Hydrogels in biology and medicine: from molecular principles to bionanotechnology. Adv Mater 18:1345-1360. https://doi.org/10.1002/adma.200501612

8. Xiaowi L, Eleni K, Xiaoyan L, Ning Z, Xuejun W (2012) Engineering neural stem cell fates with hydrogel design for central nervous system regeneration. Prog Polym Sci 37:1105-1129. https://doi.org/10.1016/j.progpolymsci.2012.02.004

9. Hubert T, Grimal S, Carroll P, Fichard-Carroll A (2009) Collagens in the developing and diseased nervous system. Cell Mol Life Sci 66:1223-1238. https://doi.org/10.1007/s00018008-8561-9

10. Phillips JB, Bunting SC, Hall SM, Brown RA (2005) Neural tissue engineering: a selforganizing collagen guidance conduit. Tissue Eng 11:1611-1617. https://doi.org/10.1089/ten. 2005.11.1611

11. Hemshekhar MM, Thushara R, Chandranayaka S, Sherman L, Kemparaju K, Girish K (2016) Emerging roles of hyaluronic acid bioscaffolds in tissue engineering and regenerative medicine. Int J Biol Macromol 86:917-928. https://doi.org/10.1016/j.ijbiomac.2016.02.032

12. Bhang S, Lee T, Lim J, Han A, Cho C (2009) The effect of the controlled release of nerve growth factor from collagen gel on the efficiency of neural cell culture. Biomaterials 30:126-132. https://doi.org/10.1016/J.BIOMATERIALS.2008.09.021

13. Xu T, Molnar P, Gregory C, Das M, Boland T, Hickman J (2009) Biomaterials 30:4377-4383. https://doi.org/10.1016/J.BIOMATERIALS.2009.04.047

14. Reetz J, Hildebrandt S, Schmidt A, Meier C, Herchenroder O et al (2016) Novel subventricular zone early progenitor cell-specific adenovirus for in vivo therapy of central nervous system disorders reinforces brain stem cell heterogeneity. Brain Struct Funct 221:2049-2059. https:// doi.org/10.1007/s00429-015-1025-8

15. Pettikiriarachchi JTS, Parish CL, Shoichet MS, Forsythe JS, Nisbet DR (2010) Biomaterials for brain tissue engineering. Aust J Chem 63:1143-1154. https://doi.org/10.1071/CH10159

16. Protasoni M, Sangiorgi S, Cividini A, Culuvaris GT, Tomei G, Dell'Orbo C, Raspanti M, Balbi S, Reguzzoni M (2011) The collagenic architecture of human dura mater. J Neurosurg 114:1723-1730. https://doi.org/10.3171/2010.12.JNS101732 
17. Antoine EE, Vlachos PP, Rylander MN (2014) Review of collagen I hydrogels for bioengineered tissue microenvironments: characterization of mechanics, structure, and transport. Tissue Eng Part B Rev 20:683-696. https://doi.org/10.1089/ten.TEB.2014.0086

18. Khaing ZZ, Schmidt CE (2012) Advances in natural biomaterials for nerve tissue repair. Neurosci Lett 519:103-114. https://doi.org/10.1016/j.neulet.2012.02.027

19. Brännvall K, Bergman K, Wallenquist U, Svahn S, Bowden T, Hilborn J et al (2007) Enhanced neuronal differentiation in a three-dimensional collagen-hyaluronan matrix. J Neurosci Res 85:2138-2146. https://doi.org/10.1002/jnr.21358

20. Bozkurt A, Brook GA, Moellers S, Lassner F, Sellhaus B, Weis J et al (2007) In vitro assessment of axonal growth using dorsal root ganglia explants in a novel three-dimensional collagen matrix. Tissue Eng 13:2971-2979. https://doi.org/10.1089/ten.2007.0116

21. Blewitt MJ, Willits RK (2007) The effect of soluble peptide sequences on neurite extension on 2D collagen substrates and within 3D collagen gels. Ann Biomed Eng 35:2159-2167. https:// doi.org/10.1007/s10439-007-9389-4

22. Deister C, Aljabari S, Schmidt CE (2007) Effects of collagen I, fibronectin, laminin and hyaluronic acid concentration in multi-component gels on neurite extension. J Biomater Sci Polym Ed 18:983-997. https://doi.org/10.1163/156856207781494377

23. Kofron CM, Fong VJ, Hoffman-Kim D (2009) Neurite outgrowth at the interface of 2D and 3D growth environments. J Neural Eng 6:016002. https://doi.org/10.1088/1741-2560/6/1/016002

24. Hiraoka M, Kato K, Nakaji-Hirabayashi T, Iwata H (2009) Enhanced survival of neural cells embedded in hydrogels composed of collagen and laminin-derived cell adhesive peptide. Bioconjug Chem 20:976-983. https://doi.org/10.1021/bc9000068

25. Kim J-M, Park WH, Min B-M (2005) The PPFLMLLKGSTR motif in globular domain 3 of the human laminin-5 alpha3 chain is crucial for integrin alpha3beta1 binding and cell adhesion. Exp Cell Res 304:317-327. https://doi.org/10.1016/j.yexcr.2004.11.009

26. Edick MJ, Tesfay L, Lamb LE, Knudsen BS, Miranti CK (2007) Inhibition of integrin-mediated crosstalk with epidermal growth factor receptor/Erk or Src signaling pathways in autophagic prostate epithelial cells induces caspase-independent death. Mol Biol Cell 18:2481-2490. https://doi.org/10.1091/mbc.e06-04-0261

27. Ekblom M, Falk M, Salmivirta K, Durbeej M, Ekblom P (1998) Laminin isoforms and epithelial development. Ann N Y Acad Sci 857:194-211. https://doi.org/10.1111/j.17496632.1998.tb10117.x

28. Galliano MF, Aberdam D, Aguzzi A, Ortonne JP, Meneguzzi G (1995) Cloning and complete primary structure of the mouse laminin alpha 3 chain. Distinct expression pattern of the laminin alpha 3A and alpha 3B chain isoforms. J Biol Chem 270:21820-21826. https://doi.org/10.1074/ jbc.270.37.21820

29. Hall PE, Lathia JD, Miller NG, Caldwell MA, ffrench-Constant C (2006) Integrins are markers of human neural stem cells. Stem Cells 24:2078-2084. https://doi.org/10.1634/stemcells.20050595

30. Schmid RS, Anton ES (2003) Role of integrins in the development of the cerebral cortex. Cereb Cortex 13:219-224. https://doi.org/10.1093/cercor/13.3.219

31. Tarone G, Hirsch E, Brancaccio M, De Acetis M, Barberis L, Balzac F, Retta SF, Botta C, Altruda F, Silengo L (2000) Integrin function and regulation in development. Int J Dev Biol 44:725-731

32. Yao L, Damodaran G, Nikolskaya N, Gorman AM, Windebank A, Pandit A (2010) The effect of laminin peptide gradient in enzymatically cross-linked collagen scaffolds on neurite growth. J Biomed Mater Res A 92:484-492. https://doi.org/10.1002/jbm.a.32359

33. Lee JH, Yu H-S, Lee G-S, Ji A, Hyun JK, Kim H-W (2011) Collagen gel three-dimensional matrices combined with adhesive proteins stimulate neuronal differentiation of mesenchymal stem cells. J R Soc Interface 8:998-1010. https://doi.org/10.1098/rsif.2010.0613

34. Swindle-Reilly KE, Papke JB, Kutosky HP, Throm A, Hammer JA, Harkins AB, Willits RK (2012) The impact of laminin on 3D neurite extension in collagen gels. J Neural Eng 9:046007. https://doi.org/10.1088/1741-2560/9/4/046007 
35. Koutsopoulos S, Zhang S (2013) Long-term three-dimensional neural tissue cultures in functionalized self-assembling peptide hydrogels, matrigel and collagen I. Acta Biomater 9:5162-5169. https://doi.org/10.1016/j.actbio.2012.09.010

36. Yu H, Cao B, Feng M, Zhou Q, Sun X, Wu S et al (2010) Combinated transplantation of neural stem cells and collagen type I promote functional recovery after cerebral ischemia in rats. Anat Rec 293:911-917. https://doi.org/10.1002/ar.20941

37. Mimura T, Amano S, Yokoo S, Uchida S, Yamagami S, Usui T, Kimura Y, Tabata Y (2008) Tissue engineering of corneal stroma with rabbit fibroblast precursors and gelatin hydrogels. Mol Vis 14:1819-1828

38. Zhong J, Chan A, Morad L, Kornblum HI, Fan G, Carmichael ST (2010) Hydrogel matrix to support stem cell survival after brain transplantation in stroke. Neurorehabil Neural Repair 24:636-644. https://doi.org/10.1177/1545968310361958

39. Hoban DB, Newland B, Moloney TC, Howard L, Pandit A, Dowd E (2013) The reduction in immunogenicity of neurotrophin overexpressing stem cells after intra-striatal transplantation by encapsulation in an in situ gelling collagen hydrogel. Biomaterials 34:9420-9429. https://doi. org/10.1016/j.biomaterials.2013.08.073

40. Liang Y, Walczak P, Bulte JWM (2013) The survival of engrafted neural stem cells within hyaluronic acid hydrogels. Biomaterials 34:5521-5529. https://doi.org/10.1016/j.biomaterials. 2013.03.095

41. Nakaji-Hirabayashi T, Kato K, Iwata H (2013) In vivo study on the survival of neural stem cells transplanted into the rat brain with a collagen hydrogel that incorporates laminin- derived polypeptides. Bioconjug Chem 24:1798-1804. https://doi.org/10.1021/bc400005m 